\title{
Interacting Generalised Cosmic Chaplygin gas in Loop quantum cosmology: A singularity free universe
}

\author{
Ratul Chowdhury 1 * \\ * Department of Chemical Engineering, Jadavpur University, Kolkata-700 032, India.
}

\author{
Prabir Rudra $2 \nmid$
}

$\dagger$ Department of Mathematics, Bengal Engineering and Science University, Shibpur, Howrah-711 103, India.

\begin{abstract}
In this work we investigate the background dynamics when dark energy is coupled to dark matter with a suitable interaction in the universe described by Loop quantum cosmology. Dark energy in the form of Generalised Cosmic Chaplygin gas is considered. A suitable interaction between dark energy and dark matter is taken into account in order to at least alleviate (if not solve) the cosmic coincidence problem. The dynamical system of equations is solved numerically and a stable scaling solution is obtained. A significant attempt towards the solution of the cosmic coincidence problem is taken. The statefinder parameters are also calculated to classify the dark energy model. Graphs and phase diagrams are drawn to study the variations of these parameters. It is seen that the background dynamics of Generalised Cosmic Chaplygin gas is completely consistent with the notion of an accelerated expansion in the late universe. From the graphs, generalised cosmic Chaplygin gas is identified as a dark fluid with a lesser negative pressure compared to Modified Chaplygin gas, thus supporting a 'No Big Rip' cosmology. It has also been shown that in this model the universe follows the power law form of expansion around the critical point, which is consistent with the known results. Future singularities that may be formed in this model as an ultimate fate of the universe has been studied in detail. It was found that the model is completely free from any types of future singularities.
\end{abstract}

\section{Introduction}

At the turn of the last century observations from Ia Supernova confirmed that our universe is suffering from an accelerated expansion [1, 2]. In the quest of finding a suitable model for the expanding universe, Cosmologists started to investigate the root cause that is triggering this expansion. Fundamentally, we were to modify Einstein's equation either by modifying the left hand side, i.e., modifying the idea of Einstein gravity or to modify the right hand side which immediately speculate the nature of the matter inside the universe. If our Universe is filled up by some invisible fluid having a large negative pressure then it violates the strong energy condition i.e. $\rho+3 p<0$. Due to its invisible nature this energy component is aptly termed as dark energy (DE) $\underline{3}$.

With the introduction of DE, the search began for different candidates that can effectively play the role of DE. DE represented by a scalar field 3 is often called quintessence. Not only scalar field but also there are other Dark fluid models like Chaplygin gas which plays the role of DE very efficiently. The earliest form of this was known as pure Chaplygin gas [4, 5]. Extensive research saw pure Chaplygin gas modify into generalized Chaplygin gas [6, 7, 8, 9, 10, Finally modified Chaplygin gas (MCG) came into existence [11, 12. Dynamics of MCG in Loop quantum cosmology was studied by Jamil et al [13. Dynamics of MCG in Braneworld was studied by Rudra et al [14.

Currently, we live in a special epoch where the densities of DE and dark matter(DM) are comparable, although they have evolved independently from different mass scales. This is known as the famous cosmic coincidence problem. till date several attempts have been made to find a solution to this problem [15, 16, 17, 18, 19, 20, 21, 22, 23, 24, 25. A suitable interaction between DE and DM is the best possible tool to find an effective solution to this problem. It is obvious that there has been a transition from a matter dominated universe to dark energy dominated universe, by

\footnotetext{
${ }^{1}$ r.chowdhury.024@gmail.com

2 prudra.math@gmail.com

${ }^{3}$ in the presence of a scalar field the transition from a universe filled with matter to an exponentially expanding universe is justified
} 
exchange of energy at an appropriate rate. Now the expansion history of the universe as determined by the supernovae and CMB data [21, 22] bounds us to fix the decay rate in such a way that it is proportional to the present day Hubble parameter. Keeping the above fact in mind cosmologists all over the world have studied and proposed a variety of interacting DE models [26, 27, 28, 29, 30, 31, 32.

In 2003, P. F. Gonzalez-Diaz [33] introduced the generalized cosmic Chaplygin gas (GCCG) model. The speciality of the model being that it can be made stable and free from unphysical behaviours even when the vacuum fluid satisfies the phantom energy condition. In the previous studies related to DE corresponding to phantom era Big-Rip is essential, as the time gradient of scale-factor blows to infinity in finite time. For the first time P. F. Gonzalez-Diaz including the GCCG model showed that Big Rip, i.e., singularity at a finite time is totally out of question. Hence in such models there is no requirement for evaporation of black hole to zero mass. The Equation of state (EOS) of this model is

$$
p=-\rho^{-\alpha}\left[C+\left\{\rho^{(1+\alpha)}-C\right\}^{-\omega}\right]
$$

where $C=\frac{A}{1+\omega}-1$, with A being a constant that can take on both positive and negative values, and $-\mathcal{L}>\omega>0, \mathcal{L}$ being a positive definite constant, which can take on values larger than unity. GCCG can explain the evolution of the universe starting from the dust era to $\Lambda C D M$, radiation era, matter dominated quintessence and lastly phantom era 34.

As we have stated earlier, modifying the right hand side of Einstein's equation was not the only way to explain the increase in the rate of expansion of the universe. The gravity part on the left hand side of Einstein's equation can also be modified to demonstrate the present day universe. In this perspective Loop quantum gravity (LQG) was introduced and Loop quantum cosmology (LQC) was developed. In this work we consider LQG as the modified gravity theory. Our main aim of this work is to examine the nature of the different physical parameters for the universe around the stable critical points in LQC model in presence of GCCG. Impact of any future singularity caused by the DE in LQC model will be studied as well.

This paper is organized as follows: Section 2 comprises of the basic equations of the LQC model. Section3 deals with the dynamical system analysis of GCCG in LQC model. In Section 4 we present a complete graphical representation of phase plane analysis. In Section 5 we study the future singularities, followed by some concluding remarks in section 6 .

\section{The Model: Loop Quantum Gravity}

In recent years, loop Quantum Gravity (LQG) has evolved as an outstanding effort to describe the quantum effect of our universe [35, 36. LQG is a theory trying to quantize the gravity with a non-perturbative and background independent method. The theory and principles of LQG when applied in the cosmological framework creates a new theoretical framework of Loop Quantum Cosmology(LQC) [37, 38, 39]. In this theory, classical space-time continuum is replaced by a discrete quantum geometry. The effect of LQG can be described by the modification of Friedmann equation by adding a term quadratic in density. In LQC, the non-perturbative effects lead to correction term $\frac{\rho_{T}^{2}}{\rho_{1}}$ to the standard Friedmann equation. With the inclusion of this term, the universe bounces quantum mechanically as the matter energy density reaches the level of $\rho_{1}$ (order of Plank density).

\subsection{Basic equations of Loop quantum gravity model}

Recently the model of DE has been explored in the framework of LQC. The cosmological evolution in LQC has been widely studied for quintessence and phantom DE models [40. The modified Friedmann equation for Loop Quantum Cosmology is given by 29, 41, 42,

$$
H^{2}=\frac{\rho_{T}}{3}\left(1-\frac{\rho_{T}}{\rho_{1}}\right)
$$

Here $\rho_{1}=\sqrt{3} \pi^{2} \gamma^{3} G^{2} \hbar$ is the critical loop quantum density and $\gamma$ is the dimensionless Barbero-Immirzi parameter. $\rho_{T}=\rho_{m}+\rho_{E}$ represents the total cosmic energy density, which is a sum of energy density of DM $\left(\rho_{m}\right)$ and the energy density of $\mathrm{DE}\left(\rho_{E}\right)$.

As in the present problem the interaction between DE in the form of GCCG and pressureless DM has been taken into account. For interacting GCCG and DM the energy balance equation will be

$$
\dot{\rho}_{g c c g}+3 H\left(1+w_{g c c g}\right) \rho_{g c c g}=-Q, \quad \text { for } G C C G
$$


and

$$
\dot{\rho}_{m}+3 H \rho_{m}=Q \text {, for the DM interacting with GCCG. }
$$

where $Q=3 b H \rho$ is the interaction term, $b$ is the coupling parameter (or transfer strength) and $\rho_{T}=\rho=\rho_{g c c g}+\rho_{m}$ is the total cosmic energy density which satisfies the energy conservation equation $\dot{\rho}+3 H(\rho+p)=0$ [43, 15].

As we have lack of information about the fact, how DE and DM interact so we are not able to estimate the interaction term from the first principles. However, the negativity of $Q$ immediately implies the possibility of having negative $\mathrm{DE}$ in the early universe which is overruled by the necessity of the second law of thermodynamics to be held [44. Hence $Q$ must be positive and small. From the observational data of 182 Gold type Ia supernova samples, CMB data from the three year WMAP survey and the baryonic acoustic oscillations from the Sloan Digital Sky Survey, it is estimated that the coupling parameter between DM and DE must be a small positive value (of the order of unity), which satisfies the requirement for solving the cosmic coincidence problem and the second law of thermodynamics [45. Due to the underlying interaction, the beginning of the accelerated expansion is shifted to higher redshifts. Consequently using the Friedmann equation (2) and the conservation equation, we obtain the modified Raychaudhuri equation

$$
\dot{H}=-\frac{1}{2}(\rho+p)\left(1-2 \frac{\rho}{\rho_{1}}\right)
$$

\section{Dynamical system analysis}

In this section we plan to analyse the dynamical system. For that firstly we convert the physical parameters into some dimensionless form, given by

$$
x=\ln a, \quad u=\frac{\rho_{g c c g}}{3 H^{2}}, \quad v=\frac{\rho_{m}}{3 H^{2}}
$$

where the present value of the scale factor, $a_{0}=1$ is assumed. Using equation (11) to equation (6) we get the parameter gradients as given below:

$$
\frac{d u}{d x}=3\left[u\left(\frac{1-v}{1+v}\right)\left\{v+u\left(1+w_{g c c g}\right)\right\}-b(u+v)-u\left(1+w_{g c c g}\right)\right]
$$

and

$$
\frac{d v}{d x}=3\left[b(u+v)-v+v\left(\frac{1-v}{1+v}\right)\left\{v+u\left(1+w_{g c c g}\right)\right\}\right]
$$

Where, $w_{g c c g}$ is the EoS parameter for GCCG determined as

$$
w_{g c c g}=\frac{p_{g c c g}}{\rho_{\text {gccg }}}=\frac{(u+v)^{4}\left[-C-\left(-C+\frac{u^{2}(u+v-1)^{2} \rho_{1}^{2}}{(u+v)^{4}}\right)^{-\omega}\right]}{u^{2}(u+v-1)^{2} \rho_{1}^{2}}
$$

In the above calculations for mathematical simplicity we have considered $\alpha=1$.

\subsection{Critical points}

The critical points of the above system are obtained by putting $\frac{d u}{d x}=0=\frac{d v}{d x}$. Now this system of equation does not yield an explicit solution. So we have to investigate for a numerical solution by putting some special values to the parameters which will yield a non zero positive solution of the system of equations. Considering $\omega=-1$, we obtain the following critical values of the system.

$$
\begin{gathered}
u_{1 c}=\frac{1}{2 b}\left(\frac{1}{4}+\frac{1}{4} P-\frac{Q}{2 \sqrt{2}}\right)\left[\frac{7}{4}-\frac{1}{4} P-3 b+\frac{Q}{2 \sqrt{2}}-b\left(\frac{1}{4}+\frac{1}{4} P-\frac{Q}{2 \sqrt{2}}\right)+2\left(\frac{1}{4}+\frac{1}{4} P-\frac{Q}{2 \sqrt{2}}\right)^{2}-\left(\frac{1}{4}+\frac{1}{4} P-\frac{Q}{2 \sqrt{2}}\right)^{3}\right] \\
v_{1 c}=\frac{1}{4}+\frac{1}{4} P-\frac{Q}{2 \sqrt{2}}
\end{gathered}
$$

and

$u_{2 c}=\frac{1}{2 b}\left(\frac{1}{4}+\frac{1}{4} P+\frac{Q}{2 \sqrt{2}}\right)\left[\frac{7}{4}-\frac{1}{4} P-3 b-\frac{Q}{2 \sqrt{2}}-b\left(\frac{1}{4}+\frac{1}{4} P+\frac{Q}{2 \sqrt{2}}\right)+2\left(\frac{1}{4}+\frac{1}{4} P+\frac{Q}{2 \sqrt{2}}\right)^{2}-\left(\frac{1}{4}+\frac{1}{4} P+\frac{Q}{2 \sqrt{2}}\right)^{3}\right]$ 


$$
v_{2 c}=\frac{1}{4}+\frac{1}{4} P+\frac{Q}{2 \sqrt{2}}
$$

where $\quad P=\sqrt{1-4 b} \quad$ and $\quad Q=\sqrt{-3+\frac{5}{\sqrt{1-4 b}}-2 b-\frac{20 b}{\sqrt{1-4 b}}}$

The above two critical points correspond to the era dominated by DM and GCCG type DE and exist for $b \leq \frac{1}{4}$. For the critical points the equation of state parameter (9) of the interacting DE takes the form

$$
w_{g c c g}=\frac{\left(u_{i c}+v_{i c}\right)^{4}\left[-C-\left(-C+\frac{u_{i c}^{2}\left(u_{i c}+v_{i c}-1\right)^{2} \rho_{1}^{2}}{\left(u_{i c}+v_{i c}\right)^{4}}\right)^{-\omega}\right]}{u_{i c}^{2}\left(u_{i c}+v_{i c}-1\right)^{2} \rho_{1}^{2}}
$$

where $i=1,2$ and which holds for $\quad u_{i c}+v_{i c} \neq 1$.

\subsection{Stability around critical point}

Now we check the stability of the dynamical system (eqs. (7) and (8)) about the critical points. In order to do this, we linearize the governing equations about the critical points i.e.,

$$
u=u_{c}+\delta u \text { and } v=v_{c}+\delta v
$$

Now if we assume $f=\frac{d u}{d x}$ and $g=\frac{d v}{d x}$, then we may obtain

$$
\delta\left(\frac{d u}{d x}\right)=\left[\partial_{u} f\right]_{c} \delta u+\left[\partial_{v} f\right]_{c} \delta v
$$

and

$$
\delta\left(\frac{d v}{d x}\right)=\left[\partial_{u} g\right]_{c} \delta u+\left[\partial_{v} g\right]_{c} \delta v
$$

where

$$
\begin{aligned}
& \partial_{u} f=\left[3 \left\{-C B^{2} M^{3}\left((4+b-4 u)(u-1) u+\left(u+u^{2}(4 u-11)+b\left(u^{2}+u-1\right)\right) v+(1+u(7 u+2 b-5)) v^{2}\right.\right.\right. \\
&+\left.(4 u+b-2) v^{3}+v^{4}\right)+\frac{1}{u^{2} \rho_{1}^{2}} N^{-\omega}\left(-u^{2} B^{2} M^{3}\left(2 u^{3}(v-1)-3 u(v+1)-2 u(1+v(3+(v-1) v)) \omega\right.\right. \\
&-v\left(v^{2}-1\right)(1+2 \omega)+u^{2}\left(5+v(-5-2 v(\omega-1)) \rho_{1}^{2}+u^{4} B^{5}\left(1+2 u(v-1)+v^{2}+b(1+v)\right) \rho_{1}^{4} N^{\omega}\right. \\
&\left.\left.\left.\left.\left.+C M^{7}\left(2 u^{3}(v-1)+v-v^{3}-3 u(v+1)+u^{2}(5+v(2 v-5))\right)\left(1+C N^{\omega}\right)\right)\right)\right) \frac{1}{(v+1) B^{3}\left(C M^{4}-u^{2} B^{2} \rho_{1}^{2}\right)}\right\}\right]
\end{aligned}
$$

$$
\begin{gathered}
\partial_{v} f=\frac{1}{(1+v)^{2}} 3 N^{-\omega}\left[-\frac{2 u^{2} M^{4}}{C M^{4}-u^{2} B^{2} \rho_{1}^{2}}+\frac{1}{-C u B^{3} M^{4} \rho_{1}^{2}+u^{3} B^{5} \rho_{1}^{4}}\left\{-u^{3} B^{5}\left(b(v+1)^{2}+u(-1+2 u+v(2+v))\right)\right.\right. \\
\rho_{1}^{4} N^{w}-2 C M^{7}\left(-2+u^{3}-3 v+v^{3}+u\left(3+v^{3}\right)+u^{2}(-2+v(2+v))\right)\left(1+C N^{\omega}\right)+u B^{2} M^{3} \rho_{1}^{2}(2 u(v+1)(u+v-2) \\
(1+u(v-1)+v)(1+\omega)+C\left(4 u^{4}+b(v-1) v(v+1)^{2}+u^{3}(v(3 v+10)-7)+u^{2}\left(7+b(v+1)^{2}+v(v+2)(4 v-3)\right)\right. \\
\left.\left.\left.\left.+u\left(-4+b(v+1)^{2}(2 v-1)+v(v+1)(-5+v(v+2))\right)\right) N^{\omega}\right)\right\}\right]
\end{gathered}
$$

$$
\begin{gathered}
\partial_{u} g=3\left[b+\left\{v ( v - 1 ) N ^ { - \omega } \left(-u^{4} B^{5} \rho_{1}^{4} N^{\omega}-C M^{7}\left(u(u-3)+v-v^{2}\right)\left(1+C N^{\omega}\right)-u^{2} B^{2} M^{3} \rho_{1}^{2}\left(-3 u+u^{2}+v-v^{2}\right.\right.\right.\right. \\
\left.\left.\left.\left.-2 u \omega+2 v \omega-2 u v \omega-2 v^{2} \omega+2 C u(u+v-2) N^{\omega}\right)\right)\right\} \frac{1}{u^{2}(1+v) B^{3} \rho_{1}^{2}\left(-C M^{4}+u^{2} B^{2} \rho_{1}^{2}\right)}\right] \\
\partial_{v} g=3\left[1+b-u-2 v+\frac{2(u-1)}{(1+v)^{2}}+\left\{M ^ { 3 } N ^ { - \omega } \left(C M ^ { 4 } \left(v(v-1)\left(3 v^{2}-5\right)+u^{2}(v(v+2)-1)+u(1+v(v(4 v+3)\right.\right.\right.\right.
\end{gathered}
$$




$$
\begin{gathered}
-6)))\left(1+C N^{\omega}\right)-u^{2} B^{2} \rho_{1}^{2}\left(u-u^{2}+5 v-6 u v+2 u^{2} v-5 v^{2}+3 u v^{2}+u^{2} v^{2}-3 v^{3}+4 u v^{3}+3 v^{4}+4 v \omega-2 u v \omega-2 v^{2} \omega\right. \\
-4 v^{3} \omega+2 v^{4} \omega+C(v(v-1) \\
\left.\left.\frac{\left.\left.\left.\left(3 v^{2}-5\right)+u^{2}(v(v+2)-1+u(1+v(-6+v(4 v+3)))) N^{\omega}\right)\right)\right)}{(1+v)^{2} B^{3} \rho_{1}^{2}\left(C u M^{4}-u^{3} B^{2} \rho_{1}^{2}\right)}\right\}\right]
\end{gathered}
$$

where $B=u+v-1, \quad M=u+v, \quad N=-C+\frac{u^{2}(u+v-1)^{2} \rho_{1}^{2}}{(u+v)^{4}}$

\subsection{Nature of cosmological parameters}

We calculate the deceleration parameter $q=-1-\left(\dot{H} / H^{2}\right)$, in this model as,

$$
q=-1+\frac{3}{2}\left(1+\omega_{g c c g} \frac{\rho_{g c c g}}{\rho}\right)\left(\frac{1-2 \frac{\rho}{\rho_{1}}}{1-\frac{\rho}{\rho_{1}}}\right)
$$

Now expressing the above expression in the form of dimensionless density parameter $\Omega_{g c c g}=\frac{\rho_{g c c g}}{\rho}$, we get,

$$
q=-1+\frac{3}{2}\left(1+\omega_{g c c g} \Omega_{g c c g}\right)\left(\frac{1-2 \frac{\rho}{\rho_{1}}}{1-\frac{\rho}{\rho_{1}}}\right)
$$

From the above expression it is evident that in the limit $\rho_{1} \rightarrow \infty$, we retrieve the result for Einstein's gravity as follows,

$$
q_{E G}=-1+\frac{3}{2}\left(1+\omega_{g c c g} \Omega_{g c c g}\right)
$$

Now assuming $\frac{\rho}{\rho_{1}}=\epsilon \sim O(1)$ and using the transformations of equation (6), we get,

$$
q=-1+\frac{3}{2}\left(1+\frac{u \omega_{g c c g}}{u+v}\right)\left(\frac{1-2 \epsilon}{1-\epsilon}\right)
$$

Since a physically acceptable solution corresponds to both the critical points, such that $(u, v) \rightarrow\left(u_{i c}, v_{i c}\right), \quad i=1,2$. Hence from equation (23) we get,

$$
q_{c}=-1+\frac{3}{2} Z, \quad \text { where } \quad Z=\left(1+\frac{u_{i c} \omega_{g c c g}}{u_{i c}+v_{i c}}\right)\left(\frac{1-2 \epsilon}{1-\epsilon}\right), \quad i=1,2
$$

As special cases, we observe that for $\epsilon=\frac{1}{2}$, we have $q=-1$ while $\epsilon=1$, we have $q=-\infty$ if $\omega_{g c c g} \geq-\frac{1}{u_{1 c}}$.

We know that the Hubble parameter varies as

$$
H=\frac{2}{3 Z t}
$$

where we have ignored the integration constant. Integration of the above equation (25) yields,

$$
a(t)=a_{0} t^{\frac{2}{3 Z}}
$$

which, as its form suggests gives a power law form of expansion.

Sahni et al [46] introduced a pair of cosmological diagnostic pair $\{r, s\}$ which is known as statefinder parameters. The two parameters are dimensionless and are geometrical since they are derived from the cosmic scale factor alone. Also this pair generalizes the well-known geometrical parameters like the Hubble parameter and the deceleration parameter. The statefinder parameters are given by

$$
r \equiv \frac{\dddot{a}}{a H^{3}}, \quad s \equiv \frac{r-1}{3(q-1 / 2)} .
$$

In the LQC model, we have the following expressions of $r$ and $s$ as

$$
r_{(L Q C)}=\left(1-\frac{3 Z}{2}\right)(1-3 Z) .
$$

and

$$
s_{(L Q C)}=Z \text {. }
$$

It is interesting to note that the pair $\left\{r_{L Q C}, s_{L Q C}\right\}$ yields the $\Lambda C D M$ (cosmological constant-cold dark matter model) $\left\{r_{E G}, s_{E G}\right\}=\{1,0\}$ when $Z=0$ or $\epsilon=\frac{1}{2}$. 


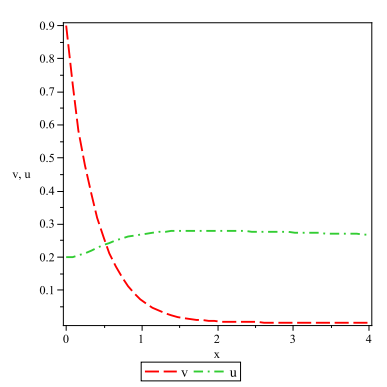

Fig. 1

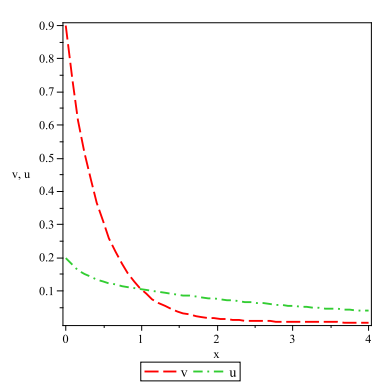

Fig. 1a

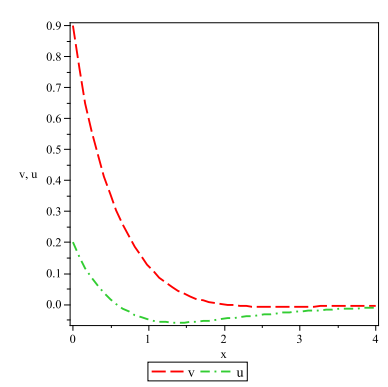

Fig. 1b

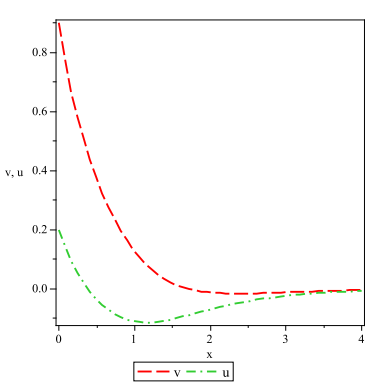

Fig. 1c

Figs.1, 1a, 1b, 1c : The dimensionless density parameters are plotted against e-folding time. The initial condition is $v(0)=0.9, u(0)=0.2$. The other parameters are fixed at $\omega=-1, C=1$ and $\rho_{1}=10$. The interactions are respectively $b=0.01,0.1,0.2$ and 0.25

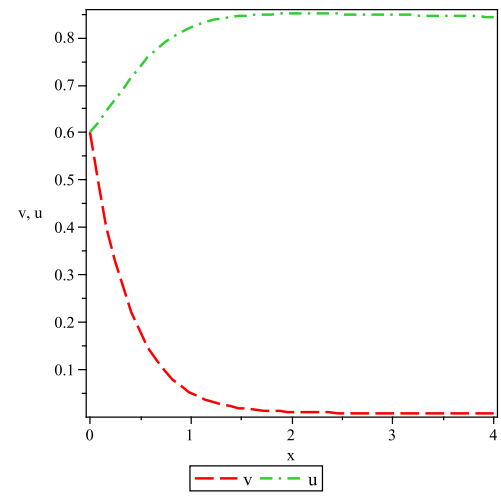

Fig. 2

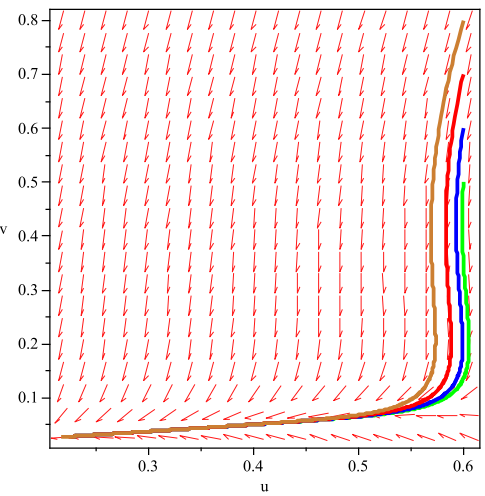

Fig. 3

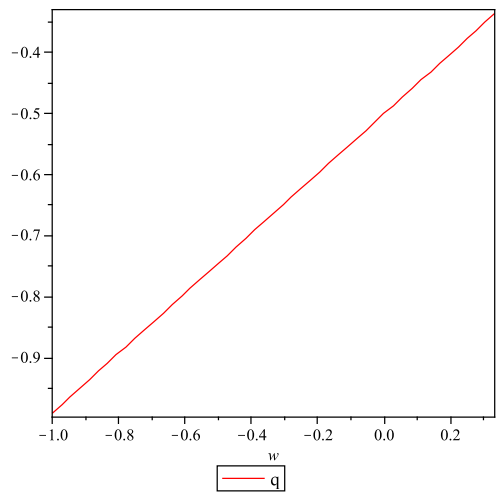

Fig. 4

Fig 2 : The dimensionless density parameters are plotted against e-folding time.The initial condition is $v(0)=0.6, u(0)=0.6$. The other parameters are fixed at $\omega=-1, b=0.01, C=1$ and $\rho_{1}=10$.

Fig 3 : The phase diagram of the parameters depicting an attractor solution. The initial conditions chosen are $v(0)=0.5, u(0)=0.6$ (green); $v(0)=0.6, u(0)=0.6$ (blue); $v(0)=0.7, u(0)=0.6($ red $) ; v(0)=$ $0.8, u(0)=0.6$ (brown). Other parameters are fixed at $\omega=-1, b=0.1, C=1$ and $\rho_{1}=10$.

Fig 4 :The deceleration parameter is plotted against the state parameter. Other parameters are fixed at $\omega=-1, b=0.01, C=1, \rho_{1}=10, \epsilon=0.4$.

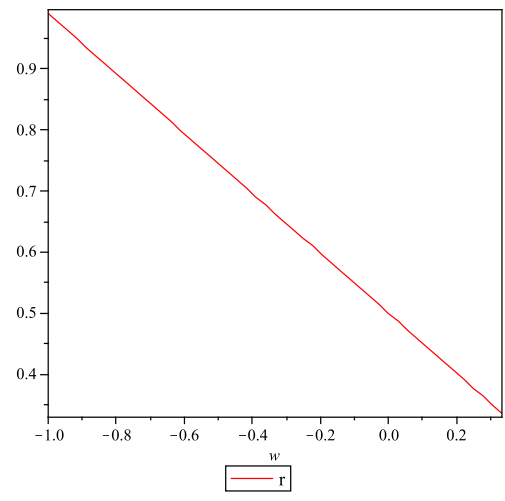

Fig. 5

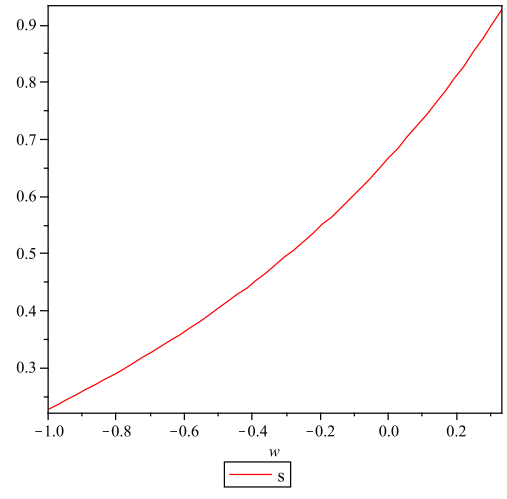

Fig. 6

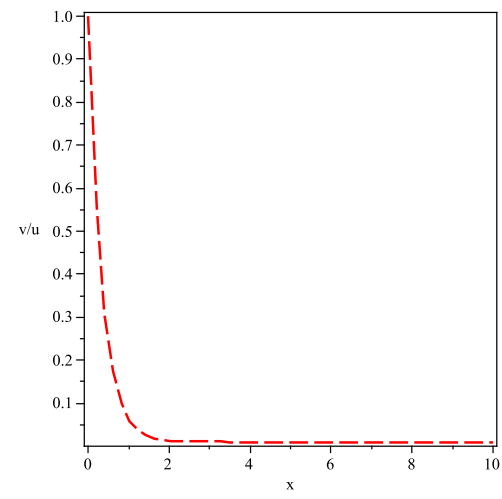

Fig. 7

Fig 5 : The statefinder parameter $r$ is plotted against the state parameter $\omega_{\text {gccg }}$. The other parameters are fixed at $\omega=-1, b=0.01, C=1, \rho_{1}=10, \epsilon=0.4$.

Fig 6 : The statefinder parameter $s$ is plotted against the state parameter $\omega_{\text {gccg }}$. The other parameters are fixed at $\omega=-1, b=0.01, C=1, \rho_{1}=10, \epsilon=0.4$.

Fig 7 : The ratio of density parameters is shown against e-folding time. The initial conditions chosen are $\mathrm{v}(0)=0.6, \mathrm{u}(0)=0.6$. The other parameters are fixed at $\omega=-1, b=0.01, C=1, \rho_{1}=10$. 


\section{Graphical Representation of the Phase plane Analysis}

Phase diagrams are drawn to determine the type of critical point obtained in this model. We discuss the results obtained in detail below:

The dimensionless density parameters $v$ and $u$ are drawn in figures $1,1 \mathrm{a}, 1 \mathrm{~b}, 1 \mathrm{c}$ and 2 . From the figures we see that $v$ decreases, and $u$ increases during evolution of the universe. This shows that the density of DM decreases while the density of DE increases as the universe evolves. So this result is consistent with the well known idea of an energy dominated universe. Figs 1, 1a, 1b and 1c are graphs depicting the same quantities with gradually increasing magnitude of interaction between GCCG and DM. It is clearly evident from the figures that with the increase in interaction between GCCG and DM, their respective density parameters ( $u$ and $v$ ) become more and more comparable to each other. Thus this interacting model probably gives the best solution to the cosmic coincidence problem. Moreover from the figs.1, 1a, 1b, 1c and 2, it is seen that the graph of DE (green curve) does not show any urgency to creep up steadily in the vertical direction. This is a striking difference from the graphs obtained by Jamil et al [13] and Rudra et al [14] in case of MCG in LQC and MCG in Braneworld respectively. So in this case it is understandable that for GCCG, the the relative domination of the DE component over DM is far lesser as compared to MCG. The possible reason may be a weaker negative pressure of GCCG compared to that of MCG. This may well be the underlying reason for GCCG supporting a 'No Big Rip' cosmology. The phase space diagram (figure 3) shows the attractor solution. The eigen values are calculated at both the critical points. At the first critical point eigen values are found to be $(0.0381055,1.52435)$. So the critical point is an unstable node. At the second critical point the eigen values are $(0.0381047,-6.09814)$. Hence the critical point is a saddle point.

Figure 4 shows the variations of the deceleration parameter, $q$ against $\omega_{g c c g}$. From figure 4 , it is evident that there is a gradual decrease in the deceleration parameter $q$, and finally in the late universe it attains negative values, which suggests that there should be an acceleration in the late universe. This result is in accordance with the various observational data of Ia supernovae and CMB data which suggests that the universe is undergoing an accelerated expansion of late. Figure 5 and 6 shows the variations of the statefinder parameters $r$ and $s$ against $\omega_{\text {gccg }}$. From these figures it can be seen that $r$ tends towards 1 and $s$ tends towards 0 . Therefore it is evident that these results tends towards the $\Lambda C D M$ model. Figure 7 shows the variation of the ratio of the density parameters $v$ and $u$ against time. From the figure it is evident that the ratio of the above parameters decreases with time. So it can be concluded that there is a relative decrease in matter density with respect to the energy density. This is again consistent with the notion of an energy dominated universe.

\section{$5 \quad$ Study of Future Singularities}

It is a well known fact that any energy dominated model of the universe is destined to result in a future singularity. The study of dynamics of an accelerating universe in the presence of DE and DM is in fact incomplete without the study of these singularities, which are the ultimate fate of the universe. It is known that the universe dominated by phantom energy ends with a future singularity known as Big Rip [47, due to the violation of dominant energy condition (DEC). But other than this there are other types of singularities as well. Nojiri et al [4] studied the various types of singularities that can result from an phantom energy dominated universe. These possible singularities are characterized by the growth of energy and curvature at the time of occurrence of the singularity. It is found that near the singularity quantum effects becomes very dominant which may alleviate or even prevent these singularities. So it is extremely necessary to study these singularities and classify them accordingly so that we can search for methods to eliminate them. The appearance of all four types of future singularities in coupled fluid dark energy, $F(R)$ theory, modified Gauss-Bonnet gravity and modified $F(R)$ Horava-Lifshitz gravity was demonstrated in [49. The universal procedure for resolving such singularities that may lead to bad phenomenological consequences was proposed. In Rudra et al 14] it has been shown that in case of Modified Chaplygin gas(MCG), both Type I and Type II singularities are possible.

\subsection{TYPE I Singularity (Big Rip singularity)}

If $\rho \rightarrow \infty,|p| \rightarrow \infty$ when $a \rightarrow \infty$ and $t \rightarrow t_{s}$. Then the singularity formed is said to be the Type I singularity.

In the present case by considering the GCCG equation of state from equation (1) we find that there is no possibility for TypeI singularity, i.e., Big Rip singularity. This is in absolute accordance with P. F. Gonzalez-Diaz who has successfully shown that by considering GCCG as the DE, Big Rip can easily be avoided, thus giving a singularity free late universe. 


\subsection{TYPE II Singularity (Sudden singularity)}

If $\rho \rightarrow \rho_{s}$ and $\rho_{s} \sim 0$, then $|p| \rightarrow-\infty$ for $t \rightarrow t_{s}$ and $a \rightarrow a_{s}$, then the resulting singularity is called the Type II singularity.

In this case we consider the equation of state for GCCG, like the previous case for our investigation. We see that if $\rho \rightarrow \rho_{s}$ and $\rho_{s} \sim 0$, then $|p| \rightarrow 0$ for $t \rightarrow t_{s}$ and $a \rightarrow a_{s}$. Hence there is no possibility of the type II singularity or the sudden singularity in case of GCCG.

\subsection{TYPE III Singularity}

For $t \rightarrow t_{s}, a \rightarrow a_{s}, \rho \rightarrow \infty$ and $|p| \rightarrow \infty$. Then the resulting singularity is Type III singularity. It is quite evident from the equation of state of GCCG that it does not support this type of singularity.

\subsection{TYPE IV Singularity}

For $t \rightarrow t_{s}, a \rightarrow a_{s}, \rho \rightarrow 0$ and $|p| \rightarrow 0$. Then the resulting singularity is Type IV singularity. This type of singularity is not supported by GCCG type DE.

As a remark, one should stress that our consideration is totally classical. Nevertheless, it is expected that quantum gravity effects may play significant role near the singularity. It is clear that such effects may contribute to the singularity occurrence or removal too. Unfortunately, due to the absence of a complete quantum gravity theory only preliminary estimations may be done.

\section{Concluding Remarks}

In this work, we have considered a combination of Generalised cosmic Chaplygin gas in Loop quantum gravity model. Our basic idea was to study the background dynamics of GCCG in detail when it is incorporated in Loop quantum gravity. Dynamical system analysis had been carried out, critical points were found and the stability of the system around those critical points was tested. Graphical analysis was done to get an explicit picture of the outcome of the work. In order to find a solution for the cosmic coincidence problem, a suitable interaction between DE and DM was considered. Figures of density parameters were drawn for different values of interaction. It was found that increase in interaction resulted in more and more comparable values of the density parameters of GCCG and DM. Since the tendency of DE domination over DM is lesser in case of GCCG compared to MCG, GCCG is identified as a dark fluid with a lesser negative pressure compared to MCG. The dynamical system of equations characterizing the system was formed and a stable scaling solution was obtained. Hence this work can be considered to be a significant one, if not the best, as far as the solution of cosmic coincidence problem is concerned. Study of future singularities had been carried out in detail. The model was investigated for all possible types of future singularities. From the above analysis we conclude that the combination of GCCG in loop quantum gravity gives a perfect singularity free model for an expanding universe undergoing a late acceleration.

\section{References}

[1] Perlmutter, S. et al. :- [Supernova Cosmology Project Collaboration], ApJ 517, 565(1999) [arXiv:astro-ph/9812133].

[2] Spergel, D. N. et al. :- WMAP Collaboration,Astron. J. Suppl 148, 175(2003) [arXiv astro-ph/0302209].

[3] Riess, A. G. et al. :- [Supernova Search Team Collaboration], ApJ 607, 665(2004)[arXiv:0402512(astro$\mathrm{ph})$ ].

[4] Kamenshchik, A., Moschella, U. and Pasquier, V. :- Phys. Lett. B 511, 265(2001).

[5] Gorini, V., Kamenshchik, A. and Moschella, U. Pasquier, V. :- [arXiv:0403062(gr-qc)].

[6] Gorini, V., Kamenshchik, A. and Moschella, U. :- Phys. Rev. D 67, 063509(2003).

[7] Alam, U., Sahni, V., Saini, T. D., Starobinsky, A.A. :- MNRAS 344 1057(2003).

[8] Bento, M. C., Bertolami, O., Sen, A. A. :- Phys. Rev. D 66, 043507(2002). 
[9] Carturan, D., Finelli, F. :-Phys. Rev. D 68 103501(2003).

[10] Barreiro, T., Sen, A.A. :- Phys. Rev. D 70 124013(2004).

[11] Benaoum, H. B.:- [arXiv:0205140(hep-th)].

[12] Debnath, U., Banerjee, A., Chakraborty, S. :- Class. Quantum. Grav. 21, 5609(2004).

[13] Jamil, M., Debnath, U. :- Astrophys. Space Sci. 333, 3 (2011).

[14] Rudra, P., Debnath, U., Biswas, R. :- Astrophys. Space Sci. DOI 10.1007/s10509-011-0967-4 [arXiv:1109.1481 (gr-qc)]

[15] del Campo, S., Herrera, R., Pavon, D. :- JCAP 0901 020(2009).[arXiv:0812.2210 (gr-qc)].

[16] Leon, G., Saridakis, E.N.:- Phys.Lett.B 693 1(2010).[ arXiv:0904.1577 (gr-qc)].

[17] Jimenez, J.B., Maroto, A.L.:- AIP Conf.Proc. 1122,107(2009).[arXiv:0812.1970 (astro-ph)].

[18] Berger, M.S., Shojae, H.:- Phys.Rev.D 73,083528(2006).[ arXiv:0601086(gr-qc)].

[19] Zhang, X.:- Mod.Phys.Lett.A 20 2575(2005).

[20] Griest, K., Phys.Rev.D66:123501(2002).[arXiv:0202052].

[21] Jamil, M., Rahaman, F.:- Eur. Phys. J. C 64 97(2009).

[22] Jamil, M., Saridakis, E.N., Setare, M.R. :- Phys. Rev. D 81 023007(2010a).

[23] Jamil, M., Saridakis, E.N. :- JCAP 07 028(2010b).

[24] Jamil, M., Farooq, M.U.:- JCAP 03 001(2010c).

[25] Jamil, M., Sheykhi, A., Farooq, M.U.:-Int. J. Mod. Phys. D 19 1831(2010d).

[26] Setare, M. R. :- Phys.Lett B 642 1(2006).

[27] Setare, M. R. :- Eur. Phys. J. C 50 991(2007c).

[28] Hu, B., Ling, Y. :- Phys. Rev. D 73 123510(2006).

[29] Wu, P., Yu, H. :- Class. Quant. Grav. 24 4661(2007).

[30] Jamil, M. :- Int. J. Theor. Phys. 49 62(2010e).

[31] Jamil, M., Momeni, D., Rashid, M.A., :- Eur. Phys. J.C 71 1711(2011) arXiv:1107.1558v1 [physics.gen-ph]].

[32] Dalal, N., Abazajian, K., Jenkins, E., Manohar, A.V., :- $\quad$ Phys. Rev. Lett $\mathbf{8 7}$ 141302(2001) arXiv:astro-ph/0105317v1].

[33] Gonzalez-Diaz, P. F. :- Phys. Rev. D 68021303 (R)(2003).

[34] Chakraborty, W., Debnath, U., Chakraborty,S. :- Grav. Cosmol. 13294 (2007) [arXiv:0711.0079(gr-qc)]

[35] Rovelli, C. :- liv. Rev. Rel. 11(1998)

[36] Ashtekar, A., Lewandowski, J. :- Class. Quantum. Grav. 21R53(2004)

[37] Ashtekar, A. :- AIP Conf. Proc. 8613(2006)

[38] Bojowald, M. :- liv. Rev. Rel. 811(2005)

[39] Ashtekar, A. et al :- Adv. Theor. Math. Phys. 7233(2003)

[40] Wu, P., Jhang, S.N., :- JCAP 06007(2008)

[41] Chen, S., Wang, B., Jing, J. :- Phys. Rev. D 78 123503(2008).

[42] Fu, X., Yu, H., Wu, P. :- Phys. Rev. D 78 063001(2008).

[43] Guo, Z.-K., Zhang, Y.-Z.:- Phys. Rev. D 71, 023501(2005).

[44] Alcaniz, J.S., Lima, J.A.S.:- Phys. Rev. D 72, 063516(2005).

[45] Feng, C. et. al.:- Phys. Lett. B 665, 111(2008). 
[46] Sahni, V. et al.:- JETP 77, 201(2003).

[47] Caldwell, R.R., Kamiokowsky,M., Weinberg,N.N. :- Phys. Rev. Lett. 91071301 (2003).

[48] Nojiri, S., Oditsov, S.D., Tsujikawa, S. :- Phys. Rev. D 71063004(2005).

[49] Nojiri, S. and Odintsov, S. D.:- Phys. Rept. 50559 (2011). 